\title{
ENTWICKLUNG EINES MULTIFUNKTIONSINSTRUMENTS FÜR DIE LAPAROSKOPISCHE CHIRURGIE
}

\author{
Timo Schröder, Dipl.-Ing. Pcter Diesing \\ Institut für Biomedizinische Technik, TU Berlin, Deutschland \\ t.schroeder@uni.de
}

\begin{abstract}
.thstract - Chirurgische Eingriffe werden zunehmend minimal-in'asiv' durchgeführt, um die Belastung der Pati'nt'n durch dic' Operution zu verringern. Die Innovation der hicr vorgestellten technischen Lösung besteht darin, dass dic cinzelne'n Instizmente, die für verschiedene Operutionsschritte erforderlich sind, in einem gemeinsamen Handgriff zusammengefasst und damit im Operationsfeld simultan und rasch austauschbar vorgehalten werden können. Durch die Verwendung von verschiedenen Aktuatoren erfolgt der Wechsel zwischen den integrierten Instrumenten automatisiert, so dass eine Einhandbedienung geviährleistet ist.
\end{abstract}

Keynords-laparoscopic instrument, endoscopic instrument, multifinctional, interchangeable operating unit

\section{Einleitung}

Für die Durchführung minimal-invasiver Operationen ist der Einsatz unterschiedlicher Instrumente unerlässlich. So sind in der Regel zumindest Scheren, Zangen und Haken mit Hochfrequenzanschlüssen für die Koagulation notwendig. Der Operateur wird normalerweise immer mit der gleichen Hand arbeiten und mit einem Halteinstrument in der anderen Hand seine Arbeit unterstützen. Dies bedeutet, dass er bei jedem Wechsel das bisherige Instrument aus dem Trokar herausziehen, bei der OP-Schwester oder Assistenten gegen das gewünschte andere austauschen und dieses wieder in den Trokar einführen muss. Hierbei gibt es einige kritische Punkte, die im folgenden kurz Betrachtet werden sollen:

1. Die Gefahr der Verunreinigung durch Keime ist außerhalb des Bauchraumes wesentlich größer.

2. Der Wechsel des Instruments nimmt Zeit in Anspruch, während der der Operateur zur Untätigkeit verdammt ist.

3. Es ist eine steril arbeitende OP-Schwester speziell zur Instrumentenverwaltung notwendig.

4. Die Kommunikation mit der OP-Schwester muss funktionieren, damit immer das gewünschte Instrument in der gewünschten Form überreicht wird.

5. Das Einführen des Instruments in den Trokar ist eine relativ hakelige Angelegenheit.

6. Der Operateur muss sein Instrument jedes Mal neu im Operationsfeld orientieren.

Durch diese Einflussfaktoren wird der Operateur insofern bei seiner Arbeit behindert, als dass er eventuell auf den Einsatz eines Instruments verzichtet, oder ihn hinauszögert, um möglichst wenige Wechsel zu haben. Dies kann die Qualität der Operation negativ beeinflussen.
Einen besonderen ökonomischen Faktor stellt der Zeitaufwand der Instrumentenwechsel dar. Durch eine Beschleunigung der Instrumentenwechsel kann kostbare OP-Zeit und eventuell sogar Personal eingespart werden.

Im Rahmen dieser Arbeit wurden in einer kurzen klinischen Studie verschiedene Ärzte bei laparoskopischen Cholesystektomien beobachtet und die benutzten Instrumente, die Anzahl der Instrumentenwechsel, sowie deren die Einsatzzeiten und die Dauer der Wechsel ausgewertet. Insgesamt wurden 12 OPs ausgewertet. Bei diesen Operationen fanden zwischen 7 und 32 Wechsel statt. Sie dauerten dabei zwischen 10 und 32 Sekunden. Durchschnittlich vergingen 24s zwischen dem Herausziehen des einen und dem ersten gezielten Einsatz des neuen Instruments. Selbst bei einem sehr erfahrenen und zügig arbeitenden Arzt lagen die Wechselzeiten im Durchschnitt bei $18 \mathrm{~s}$.

Bei einer Reduzierung der Instrumentenwechseldauer auf ca. 5s können pro Operation schnell mehrere Minuten eingespart werden. Selbst bei dem oben genannten erfahrenen Arzt hätten ca. 7\% OP-Zeit gewonnen werden können.

\section{Stand der Technik}

In der minimal-invasiven Chirurgie werden Instrumente mit Durchmessern von 3, 5 und $10 \mathrm{~mm}$ verwendet. Am verbreitetsten sind $5 \mathrm{~mm}$-Instrumente. Solche mit $10 \mathrm{~mm}$ Durchmesser werden vorwiegend für sperrige Instrumente oder Kameraoptiken verwendet. Die $3 \mathrm{~mm}$-Instrumente werden von vielen Ärzten als zu labil empfunden und dementsprechend gemieden.

Die Idee für das Multifunktionsinstrument besteht darin, mehrere der kleinen Instrumente auf der Querschnittsfläche eines Instruments mit $10 \mathrm{~mm}$ Durchmesser unterzubringen.

In der Vergangenheit wurden zwar schon mehrere Versuche unternommen, Instrumente zu entwickeln, die mehrere Funktionen in sich vereinigen, allerdings waren diese Lösungen auf manuelle Bedienung angewiesen. Dies bedeutet, dass der Benutzer zum Wechsel der Funktionen eine zweite Hand benötigt. Der Operateur ist also entweder gezwungen, das Instrument in seiner zweiten Hand loszulassen oder er benötigt die Hilfe einer zweiten Person. Dieser Umstand kann zu einer ruckartigen Bewegung führen und somit ein Verreißen des Instruments zur Folge haben. 


\section{Funktionsumfang}

Bei der hier vorgestellten Lösung wurde ein $5 \mathrm{~mm}$ Instrument und zwei $3 \mathrm{~mm}$-Instrumente ideal auf der Querschnittsfläche eines $10 \mathrm{~mm}$-Instruments verteilt.

Die beiden 3mm-Querschnitte können mit Scheren, Zangen oder Koagulationshaken bestückt werden. Hier tritt die bereits erwähnte Labilität allerdings nicht auf, da lediglich die Instrumentenspitze aus dem Führungsprofil herausragt - der restliche Schaft wird durch das Profil gestützt.

Der 5mm-Querschnitt nimmt Zusatzfunktionen, wie z.B. einen Clipapplikator auf.

Wenn man die 3 Querschnitte auf dem 10mm-Querschnitt verteilt, dann wird die Fläche nicht komplett ausgenutzt (Abbildung 1). Auf der Restfläche kann dann noch ein runder Querschnitt von bis zu 2,8mm Durchmesser, also $6,2 \mathrm{~mm}^{2}$ oder ein Profilrohr mit bis zu $18,2 \mathrm{~mm}^{2}$ Querschnittsfläche untergebracht werden. Hier kann ideal ein Spül-/Saug-Rohr untergebracht werden. Damit hat diese Lösung einen Funktionsumfang, der bisher nicht erreicht wurde.

Ein Kritikpunkt der Chirurgen an der minimal-invasiven Therapie ist, dass der Operateur durch das Instrument nur ein eingeschränktes Gefühl für das Gewebe hat. Deshalb ist das Multifunktionsinstrument aus drei separaten Instrumenten aufgebaut, die sich lediglich in einem Gehäuse befinden. Jedes besitzt seinen eigenen Auslösehebel. So wird die taktile Rückmeldung der Instrumente nicht weiter als bei konventionellen endoskopischen Instrumenten eingeschränkt. Die kleinen Instrumente mit $3 \mathrm{~mm}$ Durchmesser werden mit Zeige-, Mittel und Ringfinger geschlossen und mit dem kleinen Finger wieder geöffnet. Das $5 \mathrm{~mm}$ Instrument wird mit dem Daumen ausgelöst und mittels einer Feder wieder in Position gebracht. Hier ist die taktile Rückmeldung weniger wichtig, da der Clipapplikator lediglich fest geschlossen werden muss.

Um mit den drei Instrumenten arbeiten zu können, müssen diese axial gegeneinander verschiebbar sein, so dass sich immer nur ein Instrument zeitgleich im Operationsfeld

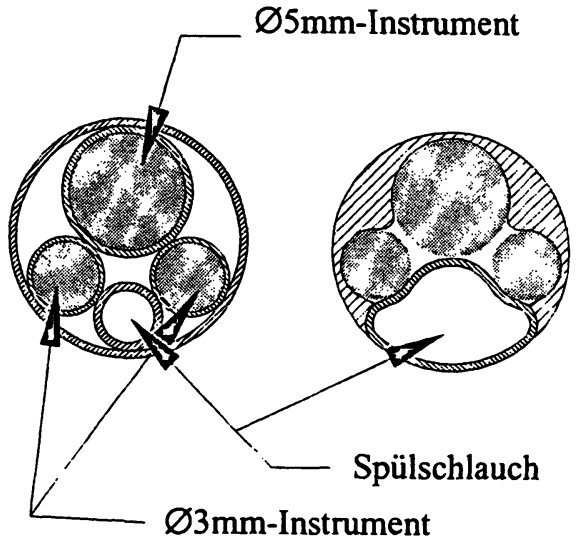

Abbildung 1: Führungskanäle mit Spülschlauch (links aus Stahlrohren, rechts mit Kunststoff-Profilrohren

befindet. Jedes der drei Instrumente ist am proximalen Ende auf einem Schlitten befestigt, der das Verschieben relativ zu dem $10 \mathrm{~mm}$-Fühnungsrohr gewährleistet. Die Übertragung der Energie zum Drehen der Instrumente erfolgt über Zahnradgetriebe ebenfalls dort. Das gewünschte Instrument ragt $30 \mathrm{~mm}$ aus dem distalen Ende des $10 \mathrm{~mm}$-Führungsquerschnitts heraus. So ist das aktive Instrument immer optimal sichtbar. Um aber eine Einhandbedienung zu ermöglichen muss das Verschieben der Instrumente durch Aktuatoren erfolgen.

Gleiches gilt für das Drehen der Instrumente. Um dem Operateur ein angenehmes Arbeiten zu ermöglichen, ist es unumgänglich, dass die Instrumente um ihre Langsachse drehbar ausgeführt werden. So kann er jedes Instrument nach seinen Bedürfnissen ausrichten und eine ergonomische Haltung einnehmen.

Bei konventionellen Instrumenten für die minimal-invasive Chirurgie wird hierzu ein Ring um den Instrumentenschaft angeordnet, an dem dieser rastend gedreht werden kann. Aufgrund der räumlichen Enge ist dies bei dem Multifunktionsinstrument nicht möglich. Deshalb werden hier auch für diese Funktion Akuatoren verwendet.

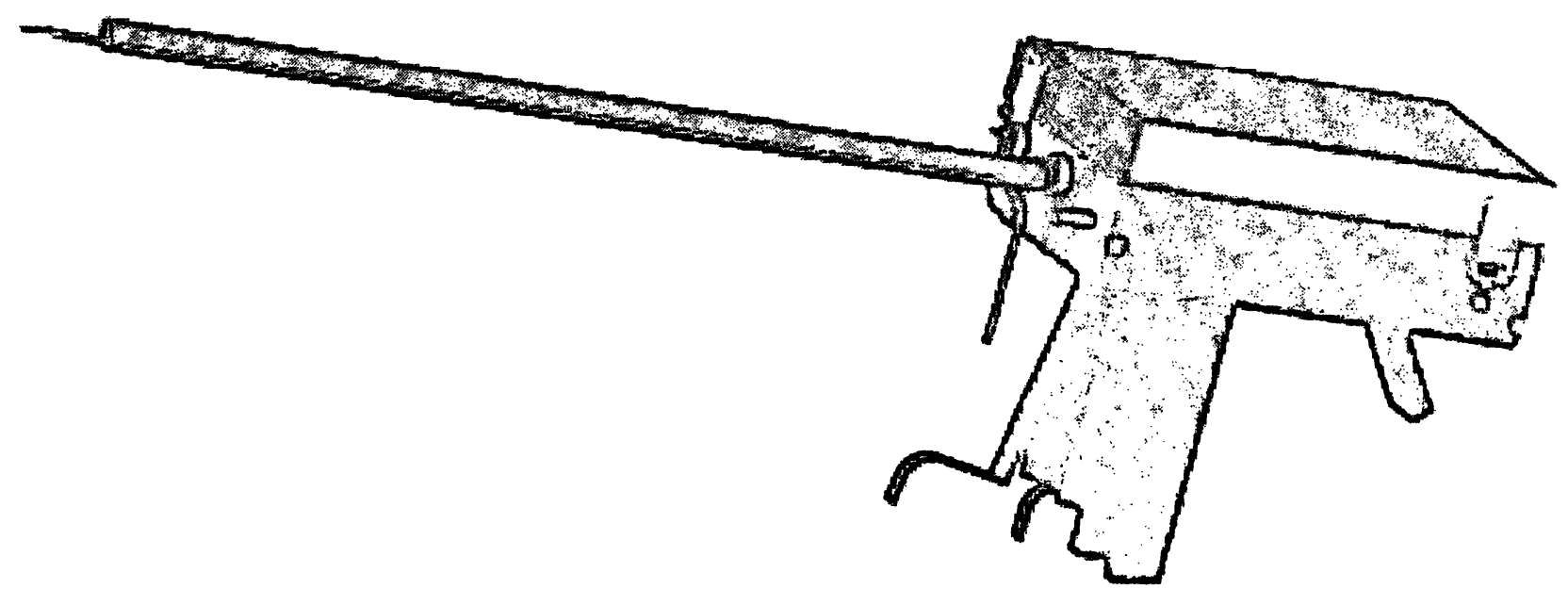

Abbildung 2: Modell des laparoskopischen Multifunktionsinstruments (Das linke 3 mm-Instrument ist in Arhe itsposstion. Der Hebel des rechten $3 \mathrm{~mm}$-Instruments ist im I Jandgriff versenkt) 


\section{Studenenwettbewerh}

Pür cin feinfühliges Justicren bzw. Verschicben der Instrumente bieten sich Mini-Schrittmotoren mit vorgeschalketen Planctengetricben an. Diese sind präzise z.l steuem, leicht und vor allem ruckfrei. Dadurch ist sichergestellt, dass der Operateur das Multifunktionsinstnument während des gesanten liinsatzes in Position halten kann.

Das (jchäuse des Multifunktionsinstruments ist zweigeteilt (Abbildung 2). In dem oberen Teil befinden sich die Motoren und Teile der Bedienclektronik. Er kann mittels Spannhebeln an dem unteren Teil befestigt werden. Der untere Teil wurde unter ergonomischen Gesichtpunkten wie ein Pistolengriff geformt auf dem die drei Instrumentenschlitten, Teile der Mechanik und die Aufnahme des Führungsrohres platziert sind. Die Hebel der 3mmInstrumente befinden sich an der Vorderseite des Handgriffs. Sie liegen nebeneinander und werden beim Zurückschieben der Schlitten im Handgriff versenkt. Der Drücker des $5 \mathrm{~mm}$ Instruments befindet sich auf dessen Rückseite und wird ebenfalls mit den Schlitten verschoben. So wird ein versehentliches Auslösen der Instrumente oder deren Verwechslung vermieden.

Die Trennung der Funktionseinheiten Motor/Elektronik und Mechanik erleichtert die Reinigung. Der untere Gehäuseteil kann mit dem gängigen Methoden (Ultraschallbad, Dampfsterilisation) gereinigt werden. Das obere Gehäuse wird durch eine sterile Einwegtasche und eine aufwendige Dichtung zum unteren Gehäuseteil vor Kontermination geschützt.

\section{Ausblick}

Zur Zeit gibt es Bestrebungen, Operationen mit Robotern durchzuführen und Patienten mittels Telemedizin zu therapieren. Der Vorteil dieser Methode liegt darin, dass der Mediziner nicht auf die räumliche Nähe zu dem Patienten angewiesen ist. Er kann seine Instrumente über große Entfernungen fernsteuern. Außerdem können die Roboter, die die Steuerbefehle umsetzen, präziser und vor allem ruhiger arbeiten, als es ein Mensch kann.

Das hier vorgestellte Multifunktionsinstrument geht bereits einen Schritt in diese Richtung. Durch die Integration der verschiedenen Aktuatoren werden Funktionen, die bislang manuell durch den Operateur durchgeführt werden mussten automatisiert.

Außerdem kann ein medizinischer Roboter nur dann sinnvoll und wirtschaftlich sein, wenn er schnell und komfortabel verschiedene Operationsschritte durchführen kann. Die Integration von vier verschiedenen Instrumenten in einem erfüllt auch diese Anforderung. Lediglich die Auslösung der Instrumente erfolgt momentan rein manuell, da dies momentan für den Operateur vor Ort angenehmer ist. Auch hier ist aber eine Ansteuerung mit Aktuatoren in Verbindung mit Kraftmessern denkbar. 